Toxicomanie et inadaptation sociale grave : perspectives subjectives de femmes en traitement quant à l'initiation et la progression de leur consommation

Drug addiction and acute social maladjustment: subjective perspectives of women in treatment in regard to the initiation and progression of their drug consumption Toxicomanía e inadaptación social grave: perspectivas subjetivas de mujeres en tratamiento con respecto a la iniciación y la progresión de su consumo de drogas

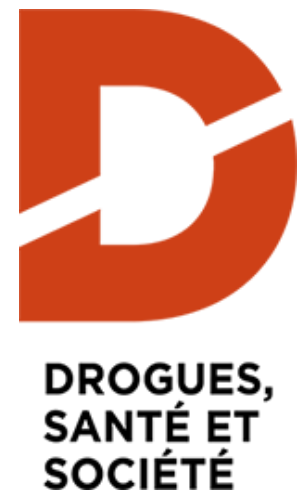

Karine Bertrand et Louise Nadeau

Volume 5, numéro 1, 2006

URI : https://id.erudit.org/iderudit/014301ar

DOI : https://doi.org/10.7202/014301ar

\section{Aller au sommaire du numéro}

\section{Éditeur(s)}

Drogues, santé et société

\section{ISSN}

1703-8839 (imprimé)

1703-8847 (numérique)

\section{Découvrir la revue}

\section{Citer cet article}

Bertrand, K. \& Nadeau, L. (2006). Toxicomanie et inadaptation sociale grave : perspectives subjectives de femmes en traitement quant à l'initiation et la progression de leur consommation. Drogues, santé et société, 5(1), 9-44. https://doi.org/10.7202/014301ar

\section{Résumé de l'article}

Des femmes présentant des problèmes graves d'inadaptation sociale concomitants à des troubles liés aux substances ont été interviewées de 5 à 8 ans après un traitement de référence. Au cours de ces entretiens, des mesures quantitatives ont été recueillies. Objectifs. Cette recherche vise à : 1) décrire, au cours de l'histoire de vie, l'initiation et la progression de la consommation d'alcool et de drogues ; 2) décrire la perspective subjective de ces femmes quant aux facteurs associés au développement de leur trajectoire de consommation d'alcool et de drogues au cours de leur vie. Méthode. Dans le cadre d'un projet de recherche plus large, 219 femmes ont été retenues sur la base de leurs résultats à l'Indice de gravité d'une toxicomanie (IGT), et ce, parmi les 2667 clients admis en traitement dans un centre public de traitement de la toxicomanie à Montréal entre 1991 et 1995. À partir de ce sous-groupe sélectionné, 21 femmes ont été interviewées dans le cadre d'une entrevue de type histoire de vie. L'IGT complète les données. Une analyse qualitative thématique du compte rendu des entrevues a été réalisée à l'aide du logiciel QSR NUD*IST. Résultats : Les récits de la toute première expérience de consommation d'alcool ou de drogues illicites sont caractérisés, à un âge précoce, par une expérience de soulagement de la tension dans un contexte familial marqué par une multiplicité de facteurs de risque, telles différentes formes de maltraitance. Le rôle de mère, l'influence d'un partenaire amoureux et la détresse psychologique constituent des éléments importants évoqués par ces femmes pour expliquer la progression de leur consommation. L'étude se termine par des recommandations cliniques. 


\title{
Toxicomanie et inadaptation sociale grave : perspectives subjectives de femmes en traitement quant à l'initiation et la progression de leur consommation
}

\author{
Karine Bertrand, \\ Professeure adjointe, service de toxicomanie du \\ Département des sciences de la santé communautaire, \\ Université de Sherbrooke \\ Louise Nadeau, \\ Professeure titulaire, Département de psychologie, \\ Université de Montréal \\ Correspondance \\ Courriel : \\ karine.bertrand@usherbrooke.ca \\ louise.nadeau.2@umontreal.ca
}




\section{Résumé}

Des femmes présentant des problèmes graves d'inadaptation sociale concomitants à des troubles liés aux substances ont été interviewées de 5 à 8 ans après un traitement de référence. Au cours de ces entretiens, des mesures quantitatives ont été recueillies. Objectifs. Cette recherche vise à : 1) décrire, au cours de l'histoire de vie, l'initiation et la progression de la consommation d'alcool et de drogues; 2) décrire la perspective subjective de ces femmes quant aux facteurs associés au développement de leur trajectoire de consommation d'alcool et de drogues au cours de leur vie. Méthode. Dans le cadre d'un projet de recherche plus large, 219 femmes ont été retenues sur la base de leurs résultats à l'Indice de gravité d'une toxicomanie (IGT), et ce, parmi les 2667 clients admis en traitement dans un centre public de traitement de la toxicomanie à Montréal entre 1991 et 1995. À partir de ce sous-groupe sélectionné, 21 femmes ont été interviewées dans le cadre d'une entrevue de type histoire de vie. L'IGT complète les données. Une analyse qualitative thématique du compte rendu des entrevues a été réalisée à l'aide du logiciel QSR NUD*IST. Résultats : Les récits de la toute première expérience de consommation d'alcool ou de drogues illicites sont caractérisés, à un âge précoce, par une expérience de soulagement de la tension dans un contexte familial marqué par une multiplicité de facteurs de risque, telles différentes formes de maltraitance. Le rôle de mère, l'influence d'un partenaire amoureux et la détresse psychologique constituent des éléments importants évoqués par ces femmes pour expliquer la progression de leur consommation. L'étude se termine par des recommandations cliniques.

Mots-clés : trajectoires, toxicomanie, femmes, récits de vie, initiation, progression 


\section{Drug addiction and acute social maladjustment: subjective perspectives of women in treatment in regard to the initiation and progression of their drug consumption}

\section{Abstract}

Women with acute social maladjustment problems related to substance abuse were interviewed, five to eight years after a reference treatment. During these meetings, quantitative measurements were gathered. Objectives: This study was designed: 1) to describe, during their life history, the initiation and progression of their alcohol and drug consumption; 2) to describe the subjective perspective of these women in regard to factors associated with the development of their alcohol and drug consumption path during their lives. Method: As part of a more extensive study, among the 2667 clients admitted to a public drug addiction centre in Montreal for treatment between 1991 and 1995, 219 women were retained on the basis of their scores in the Drug Addiction Severity Index (ASI). Within this selected sub-group, 21 women were interviewed within the scope of a life history-type interview. The Drug Addiction Severity Index completed the data. A thematic qualitative analysis of the interview report was carried out using QSR NUD*IST software. Results: The history of the very first experience of alcohol or illegal drug use were characterized, at an early age, by the experience of relieving tensions in a family situation marked by multiple risk factors, such as various forms of maltreatment. The role of the mother, the influence of a love partner and psychological distress are important components raised by these women in explaining the progression of their consumption. The study was completed by clinical recommendations.

Keywords: trajectories, drug addiction, women, life history, initiation, progression 


\section{Toxicomanía e inadaptación social grave: perspectivas subjetivas de mujeres en tratamiento con respecto a la iniciación y la progresión de su consumo de drogas}

\section{Resumen}

De cinco a ocho años después de haber seguido un tratamiento de referencia se llevaron a cabo entrevistas con mujeres que presentan problemas graves de inadaptación social originados en los problemas relacionados con el abuso de drogas. Durante estas reuniones se obtuvieron medidas cuantitativas. Objetivos: Esta investigación tiene como objetivo: 1) describir, en el curso de sus historias de vida, la iniciación y la progresión del consumo de alcohol y de drogas; 2) describir la perspectiva subjetiva de estas mujeres en lo que se refiere a los factores relacionados con el desarrollo de su trayectoria de consumo de alcohol y de drogas durante su vida. Método: En el marco de un proyecto de investigación más amplio, se eligieron 219 mujeres entre los 2667 clientes admitidos en tratamiento en un centro público de toxicomanía de Montreal entre 1991 y 1995. A partir de este subgrupo seleccionado, se entrevistó a 21 mujeres en el marco de una entrevista de tipo historia de vida. El IGT completa los datos. Con ayuda del software QSR NUD*IST, se llevó a cabo un análisis cualitativo temático de la rendición de cuentas de las entrevistas. Resultados: Los relatos del primer consumo de alcohol o de drogas ilícitas se caracterizan, en una edad precoz, por una experiencia de alivio de las tensiones en un contexto familiar marcado por una multiplicidad de factores de riesgo, tales como diferentes formas de maltrato. El papel de la madre, la influencia de un enamorado y el desamparo psicológico constituyen elementos importantes evocados por estas mujeres para explicar la progresión de su consumo de drogas. El estudio finaliza con recomendaciones clínicas.

Palabras clave: trayectoria, toxicomanía, mujeres, relatos de vida, iniciación, progresión 
Les toxicomanies surviennent fréquemment en concomitance avec d'autres problèmes graves d'inadaptation sociale, tels des troubles mentaux (pour une recension, voir Nadeau, 2001) et des problèmes légaux (Brochu \& Schneeberger, 2001). En raison de l'interaction des problèmes présentés chez ce sous-groupe de toxicomanes, leur traitement représente un défi particulier. En effet, la présence de troubles mentaux concomitants (Kessler, Nelson, McGonagle, Edlund, Frank, \& Leaf, 1996; Tomasson \& Vaglum, 1998) ainsi qu'une plus grande gravité de problèmes légaux et psychiatriques sont associées à une utilisation des services plus importante (Grella \& Joshi, 1999; Hser, Joshi, Anglin \& Fletcher, 1999; McLellan, Alterman, Metzger, Grissom, Woody, Luborsky \& O’Brien, 1994). En outre, le traitement de ces personnes est moins efficace (McLellan \& coll., 1994; Pettinati, Pierce, Belden, \& Meyers, 1999). Ces cas graves représentent une portion significative des coûts sociaux reliés à l'alcool et aux drogues en raison des nombreuses réadmissions en traitement et de leurs difficultés à être financièrement autonomes. Cet article s'intéresse au processus de changement chez ce sous-groupe de clients, lesquels représentent les cas les plus lourds.

Dans la population générale (Kessler \& coll., 1996) et dans les échantillons cliniques (Arfken, Klein, di Manza \& Schuster, 2001; Guyon \& Landry, 1996; Schuckit, \& Morissey, 1979), l'occurrence des troubles mentaux concomitants et la gravité des symptômes psychiatriques sont plus élevées chez les femmes toxicomanes que chez les hommes. Par ailleurs, particulièrement pour les femmes, les trajectoires de toxicomanie et leurs déterminants sont encore peu étudiés (Ahlstrom, Bloomfield \& Knibbe, 2001; Blume \& Zilberman, 2004; Zucker, Fitzgerald \& Moses, 1995). L'étude de la perception et des attitudes des clients concernant leur 
consommation constitue un aspect de l'examen des trajectoires qui permet de mieux saisir le processus de changement (Hser, Anglin, Grella, Longshore \& Prendergast, 1997). Par ailleurs, les données de recherche portant sur la perspective subjective des personnes toxicomanes sont rares (Mercier \& Alarie, 2000).

Les trajectoires de toxicomanie des femmes se différencient de celles des hommes. Dans la population générale ainsi que dans les populations cliniques féminines, l'abus sexuel (voir Langeland \& Hartgers, 1998; Miller \& Downs, 1995; Moncrieff \& Farmer, 1998; Wilsnack \& Wilsnack, 1995) et la dépendance à l'alcool dans la famille (Cotton, 1979; Nadeau, 1990; Vitaro, Assaad \& Carbonneau, 2004) sont les principaux facteurs de risque ayant été associés à la survenue de troubles liés aux substances psychoactives (SPA). Les résultats d'une étude récente suggèrent que l'histoire d'alcoolisme et de violence familiale durant l'enfance a un impact plus grand chez les femmes que chez les hommes au regard du développement de problèmes d'alcool et de drogues au cours de la vie adulte (Chermack, Stoltenberg, Fuller \& Blow, 2000). On note également que les femmes sont plus vulnérables à la dépression, laquelle constitue un meilleur prédicteur à la fois de la survenue et du maintien de la consommation problématique (Hartka, Johnstone, Leino, Motoyoshi, Temple \& Fillmore, 1991). De plus, les femmes sont davantage à risque de développer des problèmes liés à l'alcool que les hommes lorsque leur conjoint ou mari consomme fréquemment et que leur union est caractérisée par des insatisfactions conjugales (Hammer \& Vaglum, 1989; Kolonel \& Lee, 1981; Wilsnack, Klassen \& Schur, 1991).

L’importance du rôle du partenaire amoureux dans le développement de la trajectoire toxicomane est également documentée. Une enquête épidémiologique canadienne révèle que bon nombre de femmes rapportent que leur conjoint les a initiées à la consommation de drogues; ce modèle n'est pas courant pour les hommes (Dell \& Garabedian, 2003). Une autre étude, effectuée auprès de 277 femmes et de 365 hommes en traitement 
pour troubles liés aux substances, souligne que $60 \%$ des femmes mariées vivent avec un partenaire qui abuse de substances en comparaison à 24\% des hommes mariés (Westermeyer \& Boedicker, 2000). Les travaux de Hser et de son équipe auprès de 567 héroïnomanes en traitement de type maintien à la méthadone, en Californie, documentent également l'importance de l'influence du partenaire amoureux, tant sur le plan de l'initiation, du développement et du maintien de la dépendance (Anglin, Hser, McGlothin, 1987; Hser, Anglin \& Booth, 1987; Hser, Anglin \& McGlothin, 1987).

Quelques études qualitatives décrivent la façon dont les personnes alcooliques ou toxicomanes s'expliquent leur initiation à la consommation et leur engagement dans une trajectoire de toxicomanie. De manière générale, l’initiation à la consommation s’inscrit dans un contexte familial instable et désorganisé associé à la fois à la détresse psychologique et à la disponibilité des substances (Brunelle, Cousineau \& Brochu, 2002; Guyon, De Koninck, Morissette, Ostoj \& Marsh, 2002; Hanïnen \& Koski-Jännes, 1999). Chez les femmes, le partenaire amoureux joue un rôle significatif dans l'initiation aux drogues dures, la progression de la toxicomanie et les rechutes (Guyon \& coll., 2002; Vaglum \& Vaglum, 1987). Certains facteurs influençant la consommation de SPA sont propres aux réalités vécues par les femmes, comme le rôle du partenaire amoureux ou l'expérience de la maternité.

Force est de constater qu'aucune de ces recherches ne porte spécifiquement sur les femmes en traitement qui présentent des problèmes graves d'inadaptation sociale. Pour être en mesure de mieux aider ce sous-groupe de femmes particulièrement vulnérables, nous avons décidé d’étudier, selon leur perspective subjective, le processus de changement au cours de leur vie en portant une attention particulière aux interrelations complexes entre les divers facteurs d'influence pouvant agir à différents moments de leur trajectoire de consommation. 


\section{Objectifs de la recherche}

Cette étude vise à décrire la perspective subjective de ces femmes quant aux facteurs ou processus ayant influencé le développement de leur trajectoire toxicomane, soit l'initiation et la progression de leur consommation de substances, à différents moments au cours de leur histoire de vie. Comment décrivent-elles leur toute première expérience de consommation d'alcool ou de drogues? Qu’ont-elles vécu, ressenti ou pensé? Quel en était le contexte? Comment s'expliquent-elles la progression de la consommation de substances? Ces différentes questions ont guidé cette étude exploratoire et descriptive.

La notion de trajectoire réfère au fait que la consommation d'alcool et de drogues varie au cours de la vie selon une série de phases qui se succèdent dans le temps, de façon non linéaire (voir Mercier, 2001). Cet article s’attarde plus précisément au parcours conduisant à la survenue et au maintien de la dépendance aux substances psychoactives. Compte tenu de la complexité de ces trajectoires, nous avons choisi de présenter, dans un premier temps, la perspective subjective des participantes quant aux éléments ayant contribué à l'initiation et à la progression de leur consommation de substances au cours de leur vie. Le processus de réadaptation ou de rétablissement, caractérisé par des périodes de diminution et/ou d'arrêt de consommation à différents moments de leur vie, fait référence en soi à une autre dimension de la réalité, également fort complexe. Ces résultats ne seront donc pas abordés ici et feront plutôt l'objet d'un prochain article.

Dans cette étude, la notion de trajectoire permet d'identifier des points de repère, dans les récits de vie, facilitant l'étude en profondeur du processus de changement chez un plus petit nombre de femmes qui se prononcent sur leur propre vie. L'étude de la perspective subjective de l'acteur social quant aux sources d'influence de sa propre trajectoire est d'autant plus importante que cette subjectivité entre en interaction avec les situations 
objectives vécues. Ainsi, le vécu expérientiel, soit la façon dont l'individu s'est senti, a interprété et a réagi à divers événements ou contextes de vie, imprime un mouvement à son devenir et influence sa trajectoire (Brochu, 2006; Muchielli, 1996). Cette étude s'inscrit également dans le courant humaniste en psychologie, décrit par Carl Rogers (1951). Dans cette approche, le client est considéré comme étant l'expert de sa propre situation, d'où l'importance de s'attarder à ses perceptions subjectives pour être en mesure de mieux le comprendre et l'aider. Ainsi, cette étude qualitative peut être qualifiée de phénoménologique, au sens où elle «explore le sens manifeste des discours, elle privilégie le vécu, la perception des acteurs et de leur expérience (...) sans chercher à découvrir un sens perçu différent du sens perçu et compris par le locuteur» (Poupart, 1998).

\section{Méthodologie}

\section{Participants}

L'échantillon a été constitué à partir de critères définissant l'inadaptation sociale grave. Ce type d'échantillonnage vise à composer un groupe de participants possédant des caractéristiques qui permettent d'obtenir efficacement l'information désirée. Ce qui est recommandé en recherche qualitative pour assurer la qualité de l'information recueillie (Lessard-Hébert, 1996; Miles et Huberman, 1994). Ainsi, dans le cadre d'un projet de recherche plus large (Brochu, Bergeron, Nadeau et Landry, 1996 et 1999), 586 hommes et 219 femmes ont été sélectionnés parmi l'ensemble des 2661 usagers (822 femmes et 1839 hommes) admis entre 1991 et 1995 dans un centre public de traitement en alcoolisme et toxicomanie situé à Montréal. À leur admission en traitement, ceux-ci devaient présenter des problèmes graves et persistants à l'échelle de l'état psychologique et/ou de la situation judiciaire de 
l'Indice de Gravité d'une toxicomanie (IGT), qui sera décrit ultérieurement.

Les 219 femmes composant la clientèle la plus lourde ont en commun des problèmes plus graves dans cinq des sept sphères de vie mesurées par l'IGT en comparaison à l'ensemble des 822 femmes et des 1839 hommes admis dans ce centre public de traitement entre 1991 et 1995 . Pour chacune des sphères, les résultats sont mesurés par les scores composés de l'IGT : drogue ( $\mathrm{t}=-0,3403 ; \mathrm{P}<0,001)$; médical $(\mathrm{t}=-4,878 ; \mathrm{P}<0,0010)$; famille $(\mathrm{t}=-4,028 ; \mathrm{P}<0,00)$; état psychologique ( $\mathrm{t}=-8,272$; $\mathrm{P}<0,000)$; et emploi $(\mathrm{t}=-6,078 ; \mathrm{P}<0,000)$. La gravité des problèmes d'alcool et légaux n’est pas significativement différente dans ces deux groupes.

Parmi les 219 femmes retenues sur la base de leurs résultats à l’IGT, 39 femmes ont été rejointes en 1999 ou 2000, soit de 5 à 8 ans après la fin de leur traitement de référence. Parmi celles qui ont été rejointes, toutes celles qui ont accepté de participer à l'étude ont été interviewées, soit 21 femmes. En vue de situer l'échantillon par rapport à l'ensemble des 219 femmes répondant aux critères d'inclusion de l'étude, les scores à l'IGT des deux sous-groupes ont été comparés. En ce qui a trait à l'échantillon sous étude, lors de l'admission en traitement, il n’y a aucune différence significative entre les scores composés aux sept sphères de l'IGT et le niveau de scolarité des 21 participantes et ceux du sous-groupe de 219 femmes composant la clientèle lourde.

En moyenne, lors de l'entrevue en 2000, les 21 participantes avaient 41,9 ans et 12,1 années de scolarité. Quant à l'état civil, quatre participantes vivent avec un conjoint (toutes en union libre). Les autres sont séparées ou divorcées (6/21), veuve (1/21), ou n’ont jamais été mariées, mais ayant vécu en union libre dans le passé (8/21) ou encore jamais mariées et n’ayant jamais vécu en union libre (2/21). Quant à la source 
de revenus, la majorité vit de l'aide sociale (12/21), alors que six d'entre elles occupent un emploi et trois vivent d'une autre source de revenus, comme des prestations d'invalidité.

\section{Instruments de mesure}

Les données proviennent des deux sources suivantes : les résultats à l'Indice de gravité d'une toxicomanie (IGT) et les entrevues qualitatives de type histoire de vie.

L'IGT est une traduction de l'Addiction Severity Index (ASI) (McLellan, Luborsky et Earlen, 1980) dont la validation de la version française montre de bonnes qualités psychométriques (Bergeron, Landry, Brochu et Guyon, 1998). Ce questionnaire, présenté sous forme d'entrevue structurée, permet de mesurer la gravité des problèmes de toxicomanie à l'aide d'échelles mesurant sept sphères (alcool, drogues, santé physique, relations familiales et interpersonnelles, état psychologique, emploi et situation judiciaire). L'IGT fournit les deux séries de données répétées : celles de l'admission en traitement entre 1991 et 1995 (version longue) puis lors de l'entrevue qualitative entre 1999 et 2000 (version abrégée de recherche). On peut donc documenter l'évolution de la consommation, de 5 à 8 ans après l'admission en traitement. Les entrevues de type histoire de vie ont été réalisées au cours de deux rencontres d'environ deux heures. Une grille d'entrevue a été créée pour guider l'exploration de divers thèmes au cours de l'histoire de vie. Ces thèmes sont les variations de la consommation de substances, les événements de vie et l'expérience subjective pluridimensionnelle entourant chacune des variations de la consommation. Les données quantitatives des deux mesures de l'IGT et les récits de vie ont été mis en parallèle. Cette triangulation des sources de données augmente la fidélité et la validité des observations (Miles et Huberman, 1994; Van der Maren, 1996). 


\section{Déroulement de la recherche}

En 2000, deux entrevues de deux ou trois heures ont été réalisées auprès des participantes dans un intervalle d'environ une semaine. Une compensation financière leur a été accordée à la suite de chacune des entrevues (50 \$ en tout). Un formulaire de consentement à la recherche assurant aux participantes la confidentialité, le droit de se retirer de l'étude à tout moment et l'assurance que leur participation n'affecte en rien leur droit de recourir aux services du centre de traitement a été signé par chacune des participantes. Les participantes ont été identifiées par des prénoms fictifs et aucun détail permettant de les identifier n’est révélé dans ce texte.

\section{Analyse des données}

Le compte rendu des entrevues a été dactylographié. Une analyse thématique du contenu manifeste a été réalisée à l'aide du logiciel NUD*IST. Une table de codification a été élaborée à partir des questions de recherche. Chacun des codes a été défini dans un lexique. Les récits de vie ont été analysés de façon verticale et horizontale, c'est-à-dire que chacune des participantes a été comparée à elle-même et aux autres. Ainsi, en plus de dégager les points de convergence et de divergence dans les récits de vie, ces analyses ont permis d'examiner si certains facteurs peuvent jouer un rôle important, selon la participante, à différents moments de sa trajectoire. Les résultats à l'IGT complètent les données. Toutes ces mesures sont recommandées par Miles \& Huberman (1994) afin d'augmenter la fidélité et la validité des données.

Dans cet article, l'initiation et la progression de la consommation de substances psychoactives (SPA) réfèrent spécifiquement à l'alcool, aux drogues illicites et à la consommation de médicaments non prescrits ou abus de médicaments prescrits (c.-à-d. prendre une quantité plus grande que prescrite, 
chercher à obtenir plusieurs prescriptions de différents médecins). La cigarette et les médicaments consommés conformément à une prescription n'ont pas été considérés. L'initiation est définie comme étant la toute première expérience de consommation d'une SPA. Une progression est identifiée lorsqu'une substance est consommée plus fréquemment ou en plus grande quantité; lorsque la consommation passe d'une drogue «douce» à une drogue «dure » (c.-à-d. du cannabis à la cocaïne, de la cocaïne à l'héroïne); et lorsque le mode d'administration devient plus risqué (c.-à-d. de priser à fumer, de fumer à s’injecter). Ainsi, chez une femme qui consomme déjà du cannabis par exemple, la toute première consommation de cocaïne sera codifiée à la fois comme une initiation et comme une progression de sa consommation. Ces décisions permettent de mieux comprendre le tout premier contact de ces femmes avec une substance tout en dégageant, de façon plus globale, les éléments pouvant expliquer le développement de leur trajectoire toxicomane.

\section{Résultats}

\section{Trajectoire de consommation : données quantitatives}

\subsection{Consommation : données recueillies lors de l'admission en traitement}

L'IGT administré à l'admission en traitement entre 1991 et 1995 permet de constater que les participantes se sont initiées à plusieurs substances différentes, la plupart du temps au cours de l'adolescence. Toutes les participantes ont consommé de l'alcool, en général à partir du début de l'adolescence, soit en moyenne à 12,9 ans. Seulement trois participantes mentionnent s'y être initiées avant 12 ans. Par ailleurs, une seule mentionne n'avoir consommé aucune autre substance à part l'alcool. 
Le cannabis est la deuxième substance la plus consommée. Quatorze femmes parmi les 21 participantes s’y sont initiées en moyenne à 14,5 ans. La majorité de celles-ci, c'est-à-dire 11 sur 14, ont expérimenté des hallucinogènes durant la même période (âge moyen d'initiation de 14,9 ans). On retrouve ensuite la cocaïne, à laquelle 12 femmes se sont initiées, en moyenne à 17,8 ans. Environ la moitié des femmes (11/21) rapporte une initiation aux autres sédatifs, en moyenne à 16,1 ans. De plus, cinq de ces 11 femmes ont uniquement consommé de l'alcool et des sédatifs au cours de leur vie, ne rapportant aucune consommation d'autres drogues. Quant à l'héroïne, neuf participantes rapportent s’y être initiées en moyenne à 20,3 ans. Par ailleurs, seulement l'une d'entre elles rapporte une consommation d'héroïne à vie de plus de 6 mois.

Les substances les plus consommées pendant au moins six mois sont l'alcool, les sédatifs (autres que barbituriques), le cannabis et la cocaïne. Au moment de leur admission en traitement, les participantes rapportent avoir consommé de l'alcool jusqu'à l'intoxication pendant 14,3 ans au cours de leur vie. Ces données indiquent que l'initiation à l'alcool est précoce et implique une expérience d'intoxication lorsque consommé. Ce résultat reflète également le fait que ces femmes ont rarement expérimenté de longues périodes d’interruption de leur consommation et, lorsqu'elles consomment de l'alcool, ce n'est pas avec modération. Les autres sédatifs ont été consommés en moyenne pendant 5,1 ans. Le cannabis a été consommé en moyenne pendant 9,7 ans. On note cependant une grande variation dans les trajectoires de consommation de cannabis. Le tiers des participantes en a consommé durant moins de 6 mois au cours de leur vie, typiquement à l'adolescence. Cinq rapportent en avoir consommé durant 6 ans ou moins, également durant l'adolescence. Enfin, cinq participantes rapportent en avoir consommé pour une durée de 11 à 20 ans. 


\subsection{Consommation : données recueillies au suivi}

Les résultats à la version brève de l'IGT administrée en 2000 permettent de documenter l'évolution dans les sphères de vie. L'évolution de la gravité des problèmes entre l'admission en traitement et l'entrevue de recherche révèle que, statistiquement, l'échantillon présente, sur cinq des sept sphères mesurées, des problèmes aussi graves lors des deux temps de mesure (voir la figure 1). Par ailleurs, l'amélioration de la situation du groupe est statistiquement significative pour les problèmes d'alcool $(\mathrm{t}=3,487 ; \mathrm{P}<0,005)$ et les problèmes psychologiques ( $\mathrm{t}=3,556 ; \mathrm{P}<0,005)$.

En ce qui a trait à la consommation de substances, seulement deux participantes rapportent s'être abstenues de consommer toute substance dans les 30 jours précédant l'entrevue de recherche, quatre autres mentionnent n’avoir consommé que leurs médicaments conformément à la prescription. Onze ont consommé de l'alcool, et ce, rarement de façon modérée. En effet, elles ont toutes vécu au moins un épisode d’intoxication au cours des 30 derniers jours, le nombre moyen de jours de consommation d'alcool jusqu'à l'intoxication étant de 10,5. Parmi les 11 participantes, sept consomment toujours suffisamment d'alcool pour ressentir les effets liés à l'intoxication alors que deux autres font de même la moitié du temps. Pour les deux autres participantes qui consomment rarement de l'alcool en grande quantité, elles présentent une consommation quotidienne à une autre substance, le cannabis pour l'une et l'héroïne pour l'autre. Les autres substances ayant été consommées par les participantes dans les 30 jours précédant l'entrevue de recherche sont les suivantes : héroïne (1/21), méthadone (1/21), les autres opiacés $(7 / 21)$, les autres sédatifs (8/21), les médicaments de type antidépresseurs, antipsychotiques et lithium (11/21), la cocaïne (3/21), le cannabis (6/21) et les hallucinogènes $(1 / 21)$. 
Figure 1. Scores composés de l'IGT des participantes lors de leur admission en traitement et lors du suivi, de 5 à 8 ans après leur admission

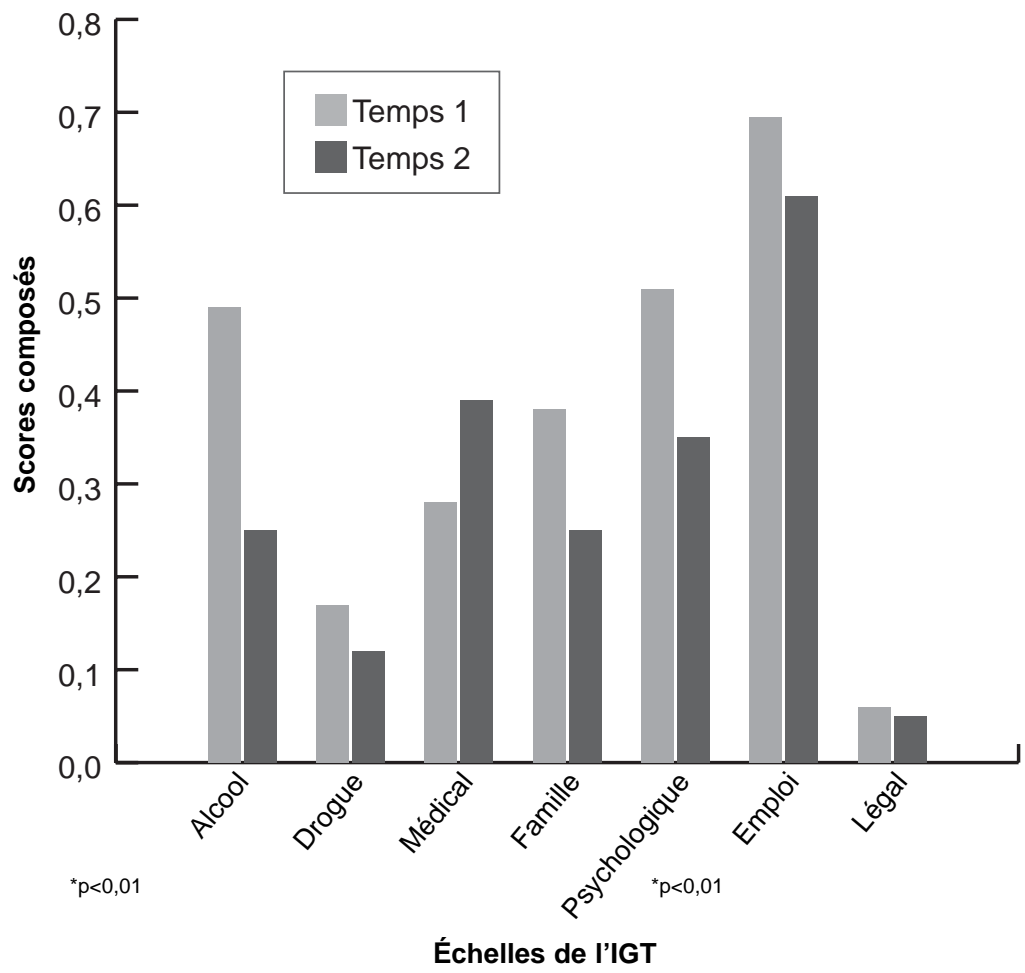




\section{Récits de vie : l'initiation et la progression}

\subsection{Initiation dans un contexte d'une multiplicité de facteurs de risque}

Nous avons demandé aux participantes de nous parler de leur enfance et de leur adolescence. Leurs récits de vie permettent d'illustrer la multiplicité des facteurs de risque vécus au cours de cette période de leur vie. Plus des deux tiers des participantes (15/21) mentionnent spontanément avoir été abusées physiquement (8/21) et/ou sexuellement (11/21) dans leur enfance ou leur adolescence. Trois expériences d'initiation à une substance se sont déroulées directement dans un contexte d'abus sexuel ou physique par une figure parentale. Deux d'entre elles se voyaient offrir de l'alcool lors des abus sexuels tandis que la mère de la troisième lui donnait de l'alcool après l'avoir battue. En outre, c'est dans un climat familial instable et désorganisé que le tiers des participantes (7/21) rapportent avoir vécu un soulagement de leur détresse lors de leur première utilisation d'une SPA, dont les effets anxiolytiques sont souvent constatés, initialement, de manière fortuite. Cette première expérience se déroule à un âge précoce : entre six et huit ans pour une, entre 12 et 16 ans pour cinq d'entre elles et à 23 ans pour la dernière. Le témoignage d’Émilie, qui a vécu dans un milieu familial dysfonctionnel (abusée sexuellement par son père alcoolique, témoin de violence conjugale), illustre cette réalité : «J’tais une fille ben ben ben, ben nerveuse (...) ça fait que, quand j'ai fumé mon premier joint (à 14 ans), là je l'ai aimé, tu peux pas savoir comment (...) parce que j'ai ressenti un calme qui s'est fait en dedans. » Par la suite, les participantes décrivent qu'elles rechercheront à nouveau à soulager leur détresse en ayant recours aux SPA.

Les récits des participantes mettent en lumière la facilité d'accès à l'alcool et aux drogues dans une sous-culture de consommation à la fois dans les réseaux familial et social. 
Ainsi, 16 participantes rapportent s’être initiées à la consommation d'une SPA avec des pairs, typiquement à une drogue illicite, entre 12 et 17 ans ; 12 participantes ont été initiées à une SPA par une figure parentale (9/21) et/ou un membre de la fratrie (5/21). Cette initiation se produit rarement dans un contexte de consommation modérée : «Ma mère, chez nous-là, c’tait traditionnel, à 13 ans, on prenait une brosse.»

\subsection{Initiation et progression : les mêmes facteurs de vulnérabilité}

Les facteurs de vulnérabilité associés à la fois à l'initiation et à la progression de la consommation se retrouvent dans quatre principaux thèmes : 1) la détresse psychologique persistante souvent associée à des moments de crise; 2) le sentiment d'échec ou d'incompétence par rapport à différentes tâches développementales, comme le départ de la maison parentale, le rôle maternel ou le rôle de travailleuse; 3) les difficultés interpersonnelles; et 4) le partenaire amoureux.

\subsubsection{Détresse psychologique et moments de crise en réaction à des stresseurs}

Les participantes structurent souvent leurs récits autour d'événements de vie. L'initiation à une SPA est reliée, pour les deux tiers des participantes, à un événement de vie, le décès d'une figure d'attachement significative étant celui le plus cité (5/21). Quant à la progression de leur consommation, toutes les participantes l'expliquent, à différents moments de leur trajectoire, par des événements de vie significatifs : déménagement (14/21); rupture amoureuse (13/21); naissance (7/21); perte d'emploi (7/21); obtention d'un nouvel emploi (7/21); perte de la garde de son enfant (6/21); et décès d'une figure d'attachement significative (6/21). Plusieurs de ces événements, comme ceux liés à l'initiation, impliquent des expériences de deuil et/ou de rejet. 


\subsubsection{Difficulté à assumer différentes tâches développementales}

Sept participantes décrivent un départ précoce de la maison familiale (15 à 18 ans) dans des circonstances conflictuelles. Le contexte de ce départ est associé à l'initiation à une nouvelle substance et/ou à la progression de leur consommation : «(...) mon père m'avait poursuivie avec des couteaux. (...) C’est là que je me suis dit que c'tait la fin de la fin, fallait que je m'en aille (...) j'tais pas prête, j'avais aucun vêtement, pas d'argent. » Caroline, 18 ans, se trouvera un emploi dans un bar et passera alors d'une consommation occasionnelle d'alcool à une consommation abusive.

La perte d’un emploi est également associée par sept participantes à la progression de leur consommation. Elles vivent alors un sentiment d'échec et perdent un élément important qui structurait leur vie, comme l'illustre le récit d'Ursula : «moi, dans le temps que j'travaillais, ça me tenait (...) fallait que j'rentre travailler le matin (...) tsé, y avait encore des restrictions (...) là [sans emploi], c'tait free for all, pis let's go. » Pour d'autres, la consommation de SPA leur permet de gérer le stress lié à l'obtention d'un nouvel emploi, dans un contexte où les substances deviennent plus accessibles.

Ces récits de vie mettent aussi particulièrement en lumière les défis importants auxquels ces femmes doivent faire face lorsqu'elles deviennent mères, souvent dans des contextes de vie difficile, comme la monoparentalité ou la présence de conflits conjugaux. Ginette explique : «J'me suis rendue compte que je n'étais pas capable de m’occuper de ma fille (...) ça marchait pas, j’tais pas prête (...).» Cinq de ces sept participantes mentionnent avoir plus tard perdu la garde de leur enfant, ce qui a suscité une progression encore plus marquée de leur consommation. La perte de la garde de leur enfant s'est produite moins d'un an après la naissance, pour quatre des six participantes ayant 
vécu cet événement. Par ailleurs, il faut noter que ces périodes de progression de la consommation sont souvent précédées d'une période d'arrêt ou de diminution de la consommation étant donné l'espoir de ces femmes de s'en sortir et de réussir à devenir une bonne mère lorsqu'elles apprennent qu'elles sont enceintes (Bertrand, Allard, Ménard et Nadeau, sous presse). Ainsi, la maternité, bien que souvent à la source de la motivation à s'engager dans une démarche de changement, est bien souvent reliée au maintien ou à l'aggravation des difficultés. Ces femmes vivent une certaine expérience de désillusion et tendent à se sentir incompétentes, incapables d'assumer leur rôle tel qu'elles l'auraient souhaité.

\subsubsection{Difficultés interpersonnelles}

Le récit des initiations à la consommation d'une SPA met en lumière les difficultés interpersonnelles des participantes. Ainsi, près de la moitié de celles-ci, comme Denise, relèvent l’importance de leur timidité ou anxiété sociale : «J'avais des problèmes à force de pas pouvoir m’exprimer, j’tais gênée (...) fait que, quand j'ai bu, tout de suite, ça m'a comme gonflée (...). J'tais capable de parler quand j'tais en boisson. » La moitié des femmes interviewées nous parlent d'isolement, d'ennui et de solitude en lien avec la progression de leur consommation.

\subsubsection{Le partenaire amoureux}

Les participantes évoquent fréquemment leur partenaire amoureux pour expliquer l'initiation et la progression de leur consommation à différents moments de leur vie. Pour ce qui est de l'initiation par un amoureux, rapportée par le tiers des participantes, celle-ci se déroule vers la fin de l'adolescence, soit de 15 à 21 ans, et est reliée à des drogues «dures » (cocaïne, hallucinogènes et méthamphétamine). La plupart du temps, elles vivent des relations amoureuses instables avec des garçons qui font partie de leur réseau d'amis consommateurs. 
Quant à la progression de la consommation d'alcool et de drogues, 12 participantes rapportent que plusieurs partenaires amoureux (en moyenne : 2,5), à différents moments dans leur vie, y ont contribué. Huit participantes relèvent que leur amoureux était vendeur de drogues, ce qui leur a facilité l'accès à des drogues illicites. Comme la plupart consommaient déjà des substances au moment de rencontrer leur partenaire amoureux, c'est à leur dépendance affective qu'elles attribuent la progression de leur consommation : «mon chum, c'tait comme ma bouée (...) j’me suis comme accrochée à lui. Lui, y était vendeur, c'est sûr qui avait un groupe après lui. (...) Ça me sécurisait, j'me sentais jamais attaquée» (Diane).

En ce qui a trait à la consommation d'alcool, l'influence du partenaire amoureux survient plus tard dans le récit des participantes dont plusieurs tendent à cohabiter, à différents moments de leur vie, avec des hommes alcooliques. Plusieurs décrivent que leur consommation est influencée par celle de leur conjoint, par un effet d'entrainement : «Ben là, ma consommation a augmenté selon lui comment il buvait. (...) Il avait un problème de boisson sûrement,(...) mais je savais même pas c'tait quoi un problème de boisson à ce moment-là» (Caroline).

De plus, la gestion, par les SPA, des difficultés conjugales, de l'intimité et de la sexualité a également été évoquée : «Y a toujours eu beaucoup d'alcool dans mes relations. (...) ça causé beaucoup de problèmes au point de vue sexuel, l'alcool ou du sexe pour quelque chose en échange. (...) C'est fini. Pas rien que les hommes, l'alcool, toute, les hommes amènent à l'alcool, pis tatati (...)» (Ginette).

Enfin, 13 participantes rapportent qu'elles ont augmenté leur consommation, surtout d'alcool (11/13), pour gérer leur détresse liée à une rupture amoureuse. Typiquement, cette rupture survient lorsqu'elles sont à la fin de la vingtaine (moyenne d'âge $=28,5$ ans) et que la consommation d'alcool est déjà 
abusive. À la suite de la rupture, elles décrivent toutes une progression importante et rapide de leur consommation qui les mène à se désorganiser.

\subsection{Activités criminelles}

Bien que la situation des participantes soit davantage caractérisée par des problèmes de santé mentale que par des problèmes légaux, un peu plus de la moitié d'entre elles (12/21) rapporte que des activités criminelles sont reliées à la progression de leur consommation. Cette délinquance au féminin s'inscrit typiquement dans un style de vie toxicomane associé à la consommation de drogues illicites (8/12). La vente de drogues (6/21) et la prostitution (6/21) sont les délits les plus rapportés. Les participantes expliquent la progression de leur consommation par trois mécanismes : 1) l'activité criminelle leur procure des revenus qui leur donnent un accès plus facile à une SPA; 2) les pairs déviants sont associés à une sous-culture de consommation; 3) la consommation de SPA peut faciliter la pratique d'une activité criminelle, particulièrement dans les cas de prostitution.

\section{Discussion}

Les données quantitatives ainsi que les récits de vie de cette étude permettent de documenter que les participantes vivent de graves problèmes dans plusieurs sphères de leur vie lorsqu'elles entrent en traitement. On constate que ces problèmes tendent à se maintenir de 5 à 8 ans plus tard. En effet, il n'y a pas eu d'amélioration significative dans les sphères mesurant la consommation de drogues, la santé physique, les relations familiales et l'emploi. Également, moins du tiers d'entre elles arrivent à maintenir l'abstinence de toute substance alors que les autres n'arrivent pas à maintenir une consommation modérée 
d'alcool ou de drogues. Par ailleurs, malgré une certaine chronicité des difficultés éprouvées par ces femmes, on note une amélioration significative de leur situation sur les plans de la gravité des problèmes d'alcool et psychologiques. Il se peut que la puissance statistique ne soit pas suffisante pour détecter certains changements ou encore que les IGT ne permettent pas de les détecter. Néanmoins, les récits de vie permettent de valider l'existence d'une certaine chronicité des problèmes.

Cette chronicité des difficultés observée dans notre étude est cohérente avec les résultats d'un ensemble de travaux. Par exemple, dans l'étude qualitative de Castel (1994) sur les sorties de la toxicomanie, la décision de modifier la consommation est très souvent accompagnée de nombreuses rechutes qui constituent en fait un cumul d'expériences menant éventuellement à un succès, bien que ce succès ne soit jamais définitif. Par ailleurs, comment expliquer que les femmes de notre étude aient vécu des améliorations sur les plans de leurs problèmes d'alcool et psychologiques, de cinq à huit ans après le traitement de référence, sans pour autant améliorer leur situation dans les autres sphères de leur vie?

Il semble que la consommation abusive de drogues soit un facteur qui contribue à la chronicisation des difficultés. En effet, les récits de vie illustrent que les femmes qui abusent de drogues tendent à vivre une escalade rapide de leur consommation, tant en termes de quantité que de types de produits. Une polytoxicomanie s’installe et l'alcool est même parfois délaissé ou consommé de façon secondaire. La consommation de cocaïne et d'héroïne, drogues illégales coûteuses, est associée à une certaine marginalisation ainsi qu’à une sous-culture déviante. Modifier sa trajectoire de toxicomanie exige alors une modification en profondeur du style de vie, tâche d'autant plus complexe que souvent les ponts avec la famille sont complètement coupés et qu'elles ne comptent aucun ami non consommateur. Les femmes qui sont aux prises avec des problèmes d'alcool 
et de médicaments présentent un profil différent qui semble les avantager sur le plan de leur démarche de changement. Elles tendent à consommer seules et à s'isoler, cachant leur consommation à leur entourage. Par ailleurs, les effets physiologiques de l'alcool et des autres sédatifs peuvent causer et aggraver les affects anxio-dépressifs. Il est donc logique qu’une amélioration significative sur le plan des problèmes d'alcool soit liée à une amélioration sur le plan des difficultés psychologiques.

Quant à l'absence d'amélioration sur les plans de la santé physique, de l'intégration socio-professionnelle et des difficultés familiales, celle-ci remet en question les services offerts à ce type de clientèle. Se peut-il que ces femmes n'aient pas bénéficié de la variété de services et du niveau d'intégration des services requis pour optimiser leurs chances de réhabilitation? En ce sens, McLellan et ses collaborateurs (1994) soulignent que ce n’est pas tant la quantité, mais plutôt la variété et la pertinence des services offerts en fonction de l'évaluation individualisée des besoins des personnes toxicomanes qui permettent de prédire le succès thérapeutique. Pour les cliniciens et prestateurs de services, cet enjeu représente un défi particulièrement complexe lorsqu'ils souhaitent aider des personnes toxicomanes aux prises avec une inadaptation sociale grave affectant plusieurs sphères de leur vie. Aussi, il faut s'interroger sur la pertinence d'intégrer davantage les membres de l'entourage dans leur traitement pour être en mesure d'avoir un impact sur le plan des relations interpersonnelles et familiales. Plusieurs études démontrent la pertinence et l'efficacité de telles approches (voir Tremblay, Bertrand et Ménard, 2006).

Cette étude a également permis de constater que les participantes ont vécu de multiples facteurs de risque au cours de leur enfance et de leur adolescence. Plus des deux tiers d'entre elles rapportent avoir été abusées physiquement ou sexuellement et la plupart décrivent un climat familial instable et désorganisé ainsi qu'un accès facile aux SPA. C'est dans ce contexte que les 
participantes rapportent avoir vécu, souvent dès leur première consommation, un soulagement de leur détresse psychologique. On comprendra que cette réaction est inséparable de la souffrance vécue par ces petites filles, notamment à travers les divers abus qu'elles ont subis. De même, la suite de leur consommation est décrite comme un soulagement de leur détresse. Par ailleurs, plusieurs études documentent que les expériences traumatiques vécues dans l'enfance sont associées à des conséquences neurobiologiques qui affectent la capacité à gérer le stress et qui peuvent en partie expliquer le maintien à l'âge adulte de symptômes psychiatriques, tels la dépression et le syndrome de stress post-traumatique (pour une recension des écrits, voir Bremner, 2003). Ainsi, ces femmes vivent, dès leur enfance, des difficultés interpersonnelles et une instabilité émotive qui les rendent vulnérables au stress pour le reste de leur vie et, par là même, aux effets de renforcement des substances. Dans ce contexte, l'accessibilité à des services à long terme impliquant une continuité entre les différents épisodes de traitement constitue un enjeu de taille pour cette clientèle.

Il est donc peu surprenant que les facteurs de vulnérabilité associés à l'initiation de la consommation soient également reliés à la progression de celle-ci au cours de leur vie. Ces femmes expliquent effectivement la progression de leur consommation par des événements de vie ayant provoqué des moments de crise et une détresse psychologique significative. L'hypothèse d'une automédication est particulièrement plausible pour les femmes de cet échantillon. On ne peut cependant exclure que leurs propos puissent être une réinterprétation des événements après le fait, ce qui est susceptible de se produire après qu’une toxicomanie eut été constatée (Barlett, 1932). Plusieurs auteurs nous rejoignent dans cette interprétation. Kessler et ses collègues (1996) montrent que le trouble mental associé aux troubles liés aux SPA apparaît habituellement en premier, et ce, plus fréquemment chez les femmes que chez 
les hommes. Velasquez, Carabonari \& DiClemente (1999), dont l'étude documente avec des mesures validées le lien entre affects négatifs et tentation de boire, estiment que l'hypothèse d'automédication apparaît particulièrement juste pour les personnes alcooliques qui présentent des troubles mentaux concomitants. De même, Castel (1994), dans l'étude précédemment citée, en vient à concevoir la toxicomanie comme un mode d'adaptation. Les expériences passées et contemporaines des participantes, la convergence de leurs propos, les difficultés inhérentes à leurs conditions de vie, les résultats d'autres études, tout converge vers une compréhension de leur consommation comme une réponse à une souffrance intérieure.

En outre, l'abus d'alcool et de drogues contribue à leur difficulté à assumer diverses tâches développementales, comme le fait de quitter le nid familial et de réussir leur vie professionnelle et amoureuse. Ces échecs contribuent à leur tour au maintien et à l'aggravation de leur trajectoire de toxicomanie. Cela peut notamment s'expliquer par le fait que la plupart de ces femmes développent leurs problèmes de consommation précocement lors de l'adolescence. Cette étape charnière du développement permet à la personne de construire son sentiment de compétence et de développer des outils pour faire face aux multiples défis de ce stade marqué par le changement : puberté, socialisation avec les pairs, début des relations amoureuses, etc. Le recours à l'alcool et aux drogues nuit considérablement à la capacité du jeune de développer les outils qui lui seront nécessaires tout au long de sa vie pour assumer ses différentes responsabilités avec succès. La consommation de substances, dans un contexte où celles-ci sont souvent très accessibles dans l'environnement, devient un mode d'adaptation duquel découlent inévitablement des échecs souvent douloureux qui tendent à se répéter ultérieurement. Il devient difficile de revenir à une consommation modérée d'alcool et de drogues lorsque celle-ci est associée 
depuis l'adolescence à une expérience d'intoxication la plupart du temps vécue en réaction à des événements stressants.

Il est clair que le stress relié aux responsabilités parentales devient souvent insurmontable pour ces femmes qui cumulent plusieurs sources de stress et qui, de plus, tendent à être plus vulnérables et moins bien équipées pour y faire face. Guyon et son équipe (2002) ont observé des résultats similaires dans leur étude auprès de mères toxicomanes. On note la même dynamique en ce qui a trait au départ du nid familial et à l'obtention d'un nouvel emploi, lesquels ont tendance à susciter des niveaux élevés de stress dépassant la capacité d'adaptation de ces femmes.

Par ailleurs, le partenaire amoureux joue un rôle clé dans l'initiation aux drogues dures et dans la progression de leur consommation d'alcool et de drogues. L'analyse de la perception des participantes recoupe les conclusions de l'étude qualitative norvégienne de Vaglum et Vaglum (1987), réalisée auprès de 64 femmes alcooliques admises en traitement psychiatrique : 1) les problèmes d'alcool de leur partenaire sont en relation avec le développement de leurs propres problèmes d'alcool, par un effet d'entraînement; 2) la détresse engendrée par une rupture est associée à une augmentation de la consommation d'alcool. De façon plus spécifique, à partir des récits de vie, on constate un risque élevé de vivre une aggravation de la trajectoire de toxicomanie lorsqu'il y a déjà une consommation à risque et que le partenaire boit beaucoup ou encore lorsqu'il y a rupture amoureuse. On peut mettre en lien ces résultats avec le fait que la plupart de ces femmes vivent des difficultés interpersonnelles significatives. La consommation joue souvent un rôle dans la facilitation des contacts sociaux. On peut penser que le fait de choisir un partenaire amoureux qui consomme abusivement alcool et drogues permet d'éviter la tâche difficile d'établir une relation sans le recours aux substances. Ces difficultés sont amplifiées par le fait que beaucoup d'entre elles ont été abusées 
sexuellement au cours de leur enfance et de leur adolescence. De plus, il semble que le choix d'un amoureux consommateur ait pu faciliter l'accès aux substances tant recherchées alors que leur trajectoire toxicomane est déjà bien installée.

Finalement, la délinquance est un autre élément important à considérer pour bien comprendre comment leur trajectoire de toxicomanie s'est installée et aggravée. Ce facteur, identifié par environ la moitié des participantes, est surtout pertinent pour comprendre le cheminement des femmes qui éprouvent des problèmes de consommation de substances psychoactives illicites. Ces récits confirment la complexité de la relation drogues-crime soulignée par Brochu (2006). Ainsi, certaines théories en cette matière, comme celle du crime lucratif où le geste délinquant vise à se procurer la drogue qui cause la dépendance, sont pertinentes pour certaines et non pour d'autres. Il se dégage tout de même que la délinquance féminine se distingue par le fait qu'elle est très rarement au centre des récits de vie. Les femmes rapportent peu de crimes violents, les délits liés à la vente de drogues et à la prostitution étant les plus fréquents. Ainsi, de façon générale, la délinquance s’inscrit dans un style de vie déviant associé à la consommation de drogues illicites. La trajectoire de toxicomanie et la trajectoire de délinquance tendent à se renforcer mutuellement. Il n'est pas rare qu'une femme aggrave sa trajectoire de toxicomanie en s'initiant à des drogues dures avec un amoureux ou un ami délinquant. Cette aggravation de sa trajectoire de toxicomanie pourra la mener à s'initier à la prostitution pour soutenir sa dépendance aux drogues.

En somme, l'analyse de la perspective subjective des participantes permet de mieux comprendre leur expérience relativement à l'initiation et à la progression de leur consommation de SPA. Cette étude apporte un éclairage nouveau aux données déjà recueillies dans le passé, souvent dans une perspective vérificatoire plutôt qu'exploratoire. Notre étude ne permet pas 
d'établir de liens de cause à effet ni de généraliser les résultats à l'ensemble des populations cliniques toxicomanes. Celle-ci vise plutôt à donner une voix aux femmes toxicomanes qui se présentent en traitement avec des problèmes graves d'inadaptation sociale en vue de mieux comprendre le développement de leur trajectoire toxicomane et leurs difficultés à modifier leur style de vie.

Nos résultats nourrissent une réflexion quant à l'importance d'adapter nos services à cette clientèle spécifique. Ainsi, pour ces femmes, il est impératif, dans l'organisation des services et du traitement, d'adopter une perspective à long terme, en relevant les défis de la continuité et de l'intégration des services qui en découlent (Mueser, Noordsy, Drake \& Fox, 2003). Le clinicien devrait également, dans une perspective d'intervention motivationnelle (Miller \& Rollnick, 2002) et non confrontante, aider ces femmes à considérer l'abstinence comme l'objectif de changement le plus pertinent, compte tenu de leur situation. En outre, le développement de compétences, particulièrement sur les plans de la gestion des émotions et des habiletés interpersonnelles, devrait être au cœur des préoccupations cliniques afin d'aider ces femmes à mieux faire face aux situations stressantes. Dans le même sens, la gestion des responsabilités parentales est de première importance, tant pour aider ces mères vulnérables que pour protéger leurs enfants. Enfin, les interrelations entre la consommation d'alcool et de drogues et les relations conjugales passées et actuelles se doivent d'être explorées afin de mieux saisir le tableau clinique et les défis auxquels doivent faire face ces femmes dans leur lutte pour s'en sortir. 


\section{Références}

Anglin, M.D., Hser, Y-I., McGlothlin, W. (1987). «Sex differences in addict careers. 2. Becoming addicted». American Journal of Drug and Alcohol Abuse. Vol. 13(3), p. 253-280.

Arfken, C. L., Klein, C., di Menza, S., \& Schuster, C. R. (2001). «Gender differences in problem severity at assessment and treatment retention». Journal of Substance Abuse Treatment. Vol. 20, p. 53-57.

Ahlstrom, S., Bloomfield, K., \& Knibbe, R. (2001). «Gender differences in drinking patterns in nine European countries: descriptive findings». Substance Abuse. 22, p. 69-85.

Barlett, F. (1932). Remembering: a Study of Experimental and Social Psychology. Cambridge : Cambridge University Press.

Bergeron, J., Landry, M., Brochu, S., \& Guyon, L. (1998). «Les études psychométriques autour de l'ASI/IGT». [In L. Guyon, M. Landry, S. Brochu, \& J. Bergeron (Eds), L'évaluation des clientèles alcooliques et toxicomanes : l'indice de gravité d'une toxicomanie (ASI/IGT)] Québec : Les Presses de l’Université Laval, p. 31-45.

Bertrand, K. (2004). Toxicomanie et inadaptation sociale sévère chez des femmes en traitement : trajectoire et services reçus. Thèse de doctorat en psychologie, Université de Montréal.

Bertrand, K., Allard, P., Ménard, J.-M. \& Nadeau, L. (sous presse). «Parents toxicomanes en traitement : une réadaptation qui protège les enfants ?» [In C. Chamberland, S. Léveillé, \& N. Trocmé (Éds), Des enfants à protéger, des adultes à aider : deux univers à rapprocher] Sainte-Foy : Presses de l'Université du Québec.

Blume, S.B., \& Zilberman, M.L. (2004). «Addiction in women». [In M. Galanter \& H. D. Kleber (Eds), The American Psychiatric Publishing textbook of substance abuse treatment (3rd edition)] Washington, DC : American Psychiatric Publishing, Inc., p. 539-546. 
Brochu, S. (2006). Drogue et criminalité. Une relation complexe. $2^{e}$ édition. Montréal : Les Presses de l’Université de Montréal.

Brochu, S., Bergeron, J., Nadeau, L., \& Landry, M. (1996). Problèmes d'inadaptation sociale graves et persistants chez les toxicomanes. [Demande de subvention au Fonds pour la Formation de Chercheurs et l'Aide à la Recherche. Manuscrit non publié].

Brochu, S., Bergeron, J., Nadeau, L., \& Landry, M. (1999). Problèmes d'inadaptation sociale graves et persistants chez les toxicomanes : suite à la première demande. [Demande de subvention au Fonds pour la Formation de Chercheurs et l'Aide à la Recherche. Manuscrit non publié].

Brochu, S., \& Schneeberger, P. (2001). «Le traitement des toxicomanes dans un contexte de pressions judiciaires ». [In M. Landry, L. Guyon, \& S. Brochu (Eds), Impact du traitement en alcoolisme et toxicomanie. Études québécoises] Québec : Les Presses de l’Université Laval, p. 73-94.

Brunelle, N., Cousineau, M-M., \& Brochu, S. (2002). «La famille telle que vécue par des jeunes consommateurs de drogues et trajectoires types de déviance juvénile». Drogues, santé et société. Vol. 1(1), p. 1-20.

Castel, R. (1994). «Les sorties de la toxicomanie». [In A. Ogian \& P. Mignon (Eds), La demande sociale de drogues] Paris : La documentation française, p. 23-30.

Chermack, S.T., Stoltenberg, S.F., Fuller, B.F. \& Blow, F.C. (2000). «Gender differences in the development of substance-related problems: The impact of family history of alcoholism, family history of violence and childhood conduct problems ». Journal of Studies on Alcohol. Vol. 61(6), p. 845-852.

Cotton, N.S. (1979). «The familial incidence of alcoholism». Journal of Studies on Alcohol. 40, p. 89-116.

Dell, C.A., \& Garabedian, K. (2003). Canadian Community Epidemiology Network on drug use (CCENDU) : 2002 national report. Ottawa : Canadian Center on Substance Abuse. 
Grella, C.E. \& Joshi, V. (1999). «Gender differences in drug treatment careers among clients in the national drug abuse treatment outcome study ». American Journal of Drug and Alcohol Abuse. Vol. 25(3), p. 385-406.

Guyon, L., De Koninck, M., Morissette, P., Ostoj, M., \& Marsh, A. (2002). «Toxicomanie et maternité. Un parcours difficile, de la famille d’origine à la famille recréée». Drogues, santé et société. Vol. 1(1), p. 1-25.

Guyon, L. \& Landry, M. (1996). «L’abus de substances psychoactives, un problème parmi d'autres? Portrait d'une population en traitement». Psychotropes, Revue Internationale des Toxicomanies. 1, p. 61-81.

Hammer, T. et Vaglum, P. (1989). «The increase in alcohol consumption among women: a phenomenon related to accessibility or stress? A general population study». British Journal of Addiction. 84, p. 767-775.

Hänninen, V., \& Koski-Jânnes, A. (1999). «Narratives of recovery from addictive behaviours ». Addiction. Vol. 94(12), p. 1837-1848.

Hartka, E., Johnstone, B.M., Leino, E.V., Motoyoshi, M., Temple, M.T. et Fillmore, K.M. (1991). «A meta-analysis of depressive symptomatology and alcohol consumption over time». British Journal of Addiction. 86, p. 1283-1298.

Hser, Y-I., Anglin, M.D., \& Booth, M.A. (1987). «Sex differences in addict careers. 3. Addiction». American Journal of Drug and Alcohol Abuse. Vol. 13(3), p. 231-251.

Hser, Y-I., Anglin, M.D., \& McGlothlin, W. (1987). «Sex differences in addict careers. 1. Initiation of use». American Journal of Drug and Alcohol Abuse. Vol. 13(1 \& 2), p. 33-57.

Hser, Y-I., Anglin, M.D., Grella, C., Longshore, D., \& Prendergast, M.L. (1997). «Drug treatment careers. A conceptual framework and existing research findings ». Journal of substance abuse and treatment. Vol. 14(6), p. 543-558. 
Hser,Y., Joshi, V., Anglin, M.D., \& Fletcher, B.W. (1999). «Predicting posttreatment cocaine abstinence for first-time admissions and treatment repeaters ». American Journal of Public Health. Vol. 89(5), p. 666-671.

Jos, P.H., Perlmutter, M., \& Marshall, M.F. (2003). «Substance abuse during pregnancy: clinical and public health approaches». Journal of law, medecine \& ethics. Vol. 31(3), p. 340-350.

Kessler, R. C., Nelson, C. B., McGonagle, K. A., Edlund, M. J., Frank, R. G., \& Leaf, P. J. (1996). «The epidemiology of co-occurring addictive and mental disorders: implications for prevention and service utilization». American Journal of Orthopsychiatry. 66, p. 17-31.

Kolonel, L.N. et Lee, J. (1981). «Husband-wife correspondence in smoking, drinking and dietary habits». American Journal of Clinical Nutrition. 34, p. 99-104.

Lessard-Hébert, M., Goyette, G. et Boutin, G. (1996). La recherche qualitative : Fondements et pratiques, $2^{e}$ édition. Montréal : Éditions Nouvelles.

Langeland, W. et Hartgers, C. (1998). "Child sexual and physical abuse and alcoholism: A review». Journal of Studies on Alcohol. 59, p. 336-348.

McLellan, A.T., Alterman, A.I., Metzger, D.S., Grissom, G.R., Woody, G.E. Luborsky, L., \& O’Brien, C.P. (1994). «Similarity of outcome predictors across opiate, cocaine, and alcohol treatments: role of treatment services ». Journal of Consulting and Clinical Psychology. Vol. 62(6), p. 1141-1158.

McLellan, A.T., Luborsky, L., \& Earlen, F. (1980). «The Addiction Severity Index». [In E. Gottheil, A.T. McLennan, \& K.A. Druley (Éds), Substance Abuse and Psychiatric Illness] New York : Pergamon Press, p. 71-120.

Mercier, C. (2001). Addiction trajectories. Papier présenté dans le cadre du Senate Committee on Illegal Drugs, décembre 2001, Ottawa. [En ligne] [http://www.parl.gc.ca/37/1/parlbus/commbus/senate/ Com-e/ille-e/Presentation-e/mercier-e.htm]. 
Mercier, C. \& Alarie, S. (2000). «Le processus de rétablissement chez les personnes alcooliques et toxicomanes». [In P. Brisson, L'usage des drogues et toxicomanie, Volume III] Montréal : Gaëtan Morin, p. 336-349.

Miles, M.B., \& Huberman, A.M. (1994). Qualitative data analysis, second edition. Thousand Oaks, Ca : Sage Publications.

Miller, B.A. et Downs, W.R. (1995). «Violent victimization among women with alcohol problems ». [In M. Galanter (éd), Recent developments in alcoholism, volume 12: women and alcoholism] New York : Plenum press, p. 81-101.

Miller, W.R. et Rollnick, S. (2002). Motivational Interviewing. Preparing people for change. Second edition. New York : Guilford Press.

Moncrieff, J. et Farmer, R. (1998). «Sexual abuse and the subsequent development of alcohol problems ». Alcohol and Alcoholism. 33, p. 592-601.

Mucchielli, A. (1996). Dictionnaire des méthodes qualitatives en sciences humaines et sociales. Paris : Armand Colin.

Mueser, K.T., Noordsy, D.L., Drake,R.E. \& Fox, L. (2003). Integrated Treatment for dual disorders. A guide to effective practice. New York : Guilford Press.

Nadeau, L. (2001). «La cooccurrence de la toxicomanie et des autres troubles mentaux». [In M. Landry, L. Guyon, \& S. Brochu (Eds), Impact du traitement en alcoolisme et toxicomanie. Études québécoises] Québec : Les Presses de l’Université Laval, p. 45-71.

Nadeau, L. (1990). «Les problèmes liés à l'alcool chez les femmes : l'examen de l'hypothèse d'une interaction entre des facteurs de vulnérabilité et des agents déclencheurs ». Revue canadienne des sciences du comportement. 22, p. 433-444.

Pettinati, H.M., Pierce, J.D., Belden, P.P., \& Meyers, K. (1999). «The relationship of Axis II personality disorders to other known predictors of addiction treatment outcome ». American Journal of Addiction. Vol. 8(2), p. 136-147. 
Poupart, J. (1998). La recherche qualitative : diversité des champs et des pratiques au Québec. Boucherville : Gaëtan Morin.

Rogers, C. (1951). Client-centered therapy. Boston : Houghton Mifflin.

Schuckit, M. A., \& Morrissey, E. R. (1979). «Drug abuse among alcoholics women ». American Journal of Psychiatry. 136, p. 607-611.

Tomasson K, Vaglum P. (1998). «Psychiatric co-morbidity and aftercare among alcoholics: a prospective study of a nationwide representative sample». Addiction. Vol. 93(3), p. 423-431.

Tremblay, J., Bertrand, K. \& Ménard, J-M. (2005). Implication des membres de l'entourage dans le processus de réadaptation des personnes alcooliques et toxicomanes. Comité permanent de lutte à la toxicomanie, $126 \mathrm{p}$.

Vaglum, S. et Vaglum, P. (1987). «Partner Relations and the Development of Alcoholism in Psychiatric Patients ». Acta psychiatrica Scandinavica. 76, p. 499-506.

Van der Maren, J.M. (1996). Méthodes de recherche pour l'éducation, $2^{e}$ édition. Montréal : Presses de l’Université de Montréal.

Velasquez, M.M., Carbonari, J.P., \& DiClemente, C.C. (1999). «Psychiatric severity and behavior change in alcoholism: the relation of the transtheoretical model variables to psychatric distress in dually diagnosed patients». Addictive Behaviors. Vol. 24(4), p. 481-496.

Vitaro, F., Assaad, J.M. \& Carbonneau, R. (2004). Les enfants de parents affectés d'une dépendance : Bilan des connaissances et leçons pour l'intervention. Montréal : Comité permanent de lutte à la toxicomanie.

Westermeyer, J., \& Boedicker, B.A. (2000). «Course, severity, and treatment of substance abuse among women versus men ». American Journal of drug and alcohol abuse. Vol. 26(4), p. 523-535. 
Wilsnack, S.C., Klassen, A.D. et Schur, B.E. (1991). «Predicting onset and chronicity of women's problem drinking: a five-years longitudinal analysis ». American Journal of Public Health. 81, p. 305-318.

Wilsnack, S.C. et Wilsnack, R.W. (1995). «Drinking and problem drinking in US women. Patterns and recent trends». [In M. Galanter, Recent Developments in Alcoholism, volume 12, alcoholism and women] New York : Plenum Press.

Zucker, R.A., Fitzgerald, H.E., \& Moses, H.D. (1995). «Emergence of alcohol problems and the several alcoholism: a developmental perspective on etiologic theory and life course trajectory ». [In D. Cicchetti, \& D.J. Cohen, Developmental Psychopathology, volume 2: Risk, Disorder, and Adaptation] Toronto : John Wiley, \& Sons, Inc, p. 677-711. 